\title{
Rashba effect at magnetic metal surfaces
}

\author{
O. Krupin, ${ }^{1}$ G. Bihlmayer, ${ }^{2}$ K. Starke, ${ }^{1, *}$ S. Gorovikov, ${ }^{3}$ J. E. Prieto, ${ }^{1}$ K. Döbrich,,${ }^{1}$ S. Blügel, ${ }^{2}$ and G. Kaindl ${ }^{1}$ \\ ${ }^{1}$ Institut für Experimentalphysik, Freie Universität Berlin, Germany \\ ${ }^{2}$ Institut für Festkörperforschung, Forschungszentrum Jülich, Germany \\ ${ }^{3}$ MAX-Lab, Lund University, Sweden
}

(Received 28 March 2005; published 31 May 2005)

\begin{abstract}
Exchange-split two-dimensional electronic states at the magnetic $\operatorname{Gd}(0001)$ surface change their energy dispersion upon magnetization reversal owing to the Rashba effect. The Rashba parameter is found to be substantially enhanced and to change sign when an epitaxial metal-oxide surface layer is formed. The experimental observations are quantitatively described by $a b$ initio calculations giving a detailed account of the near-surface charge-density gradients that are responsible for the Rashba effect.
\end{abstract}

DOI: 10.1103/PhysRevB.71.201403

PACS number(s): 73.20.-r, 71.70.Ej, 72.25.-b, 85.75.-d

A key topic in condensed-matter research aiming at future spintronic devices is concerned with the control and manipulation of the electron spin in a two-dimensional electron gas (2DEG) without the need to apply an external magnetic field. ${ }^{1}$ Rashba had realized early on ${ }^{2}$ that this can be achieved by an electric field that acts as a magnetic field in the rest frame of a moving electron. The interaction between the spin $\mathbf{s}$ of an electron, moving with momentum $\hbar \mathbf{k}$ in an electric field oriented along the $z$ axis $\mathbf{e}_{z}$, is described by the Rashba Hamiltonian

$$
\mathcal{H}_{R}=\alpha_{R}\left(\mathbf{e}_{z} \times \mathbf{k}\right) \cdot \mathbf{s}
$$

Here, $\alpha_{R}$ is the so-called Rashba parameter, which is proportional to the electric field and depends further on the effective, material-dependent, spin-orbit coupling (SOC) strength. A precondition for the Rashba effect is structural inversion asymmetry (SIA), which naturally exists at any crystal interface or surface, and Rashba splittings have indeed been observed for surface states of $\mathrm{Au}(111)$ (Refs. 3 and 4) and other nonmagnetic surfaces., 5 Although there is a close analogy between the Rashba effect revealed by free-electron-like $(s p-)$ surface states and a 2DEG (Ref. 7), crystalline surfaces have been found to exhibit no Rashba effect without the presence of high- $Z$ elements that give rise to substantial intra-atomic SOC (Refs. 3 and 4). This indicates that the surface-potential gradient alone is not a good measure of the effective electric field that is responsible for the effect. ${ }^{3}$

Experimental studies of the Rashba effect so far have been limited to semiconductor heterostructures ${ }^{8}$ and nonmagnetic metal surfaces. This is surprising when one considers (i) the essential role of magnetic materials for spin injection in the suggested spintronic devices and (ii) the indispensible ferromagnetic/nonmagnetic interfaces (FM/ NM) in hybrid structures. ${ }^{9}$

This Communication presents an experimental investigation of two-dimensional electronic states in which exchange interaction and the Rashba effect coexist. We use the surface state of ferromagnetic Gd metal, as well as a FM/NM interface state between ferromagnetic $\mathrm{Gd}$ and a nonmagnetic metal-oxide surface layer, as examples to demonstrate that-in cases of dominant exchange interaction-the
Rashba contribution to the electron binding energy changes sign upon magnetization reversal. We also find that the Rashba parameter is substantially enhanced and reversed upon formation of the metal-oxide surface layer. These observations are quantitatively described by $a b$ initio calculations, elucidating the role which charge-density gradient and orbital character of a two-dimensional state play for size and sign of the Rashba effect in real systems.

To investigate the Rashba effect at magnetic surfaces, it is advantageous to choose a ferromagnet that contains a high- $Z$ element, like the close-packed (0001) surface of Gd metal, which has large intra-atomic SOC together with a substantial valence-band exchange splitting on the order of $0.5 \mathrm{eV}$ (Ref. 10). Angle-resolved photoemission (PE) experiments were performed using display-type electron analyzers at the I-311 undulator beamline at MAX-Lab and the BUS beamline at BESSY II. The overall energy resolution was set to $30 \mathrm{meV}$ full width at half maximum (FWHM), and the angular resolution to $0.5^{\circ} \cdot \operatorname{Gd}(0001)$ films were prepared in situ by metal vapor deposition on a W(110) single crystal. The film thickness was $10 \mathrm{~nm}$ as determined by a quartz balance. During evaporation the pressure in the UHV chamber rose from 5 $\times 10^{-11}$ to $5 \times 10^{-10}$ mbar. The epitaxial surface monoxide layer was prepared on $\mathrm{Gd}(0001)$ according to the recipe given in Ref. 11 with a two-Langmuir oxygen exposure; it was controlled in situ by PE and low-energy electron diffraction (LEED). The PE spectra were recorded using remanently magnetized samples in the experimental geometry of Fig. 1(c).

At the ferromagnetic $\mathrm{Gd}(0001)$ surface, only the majority component $S_{\uparrow}$ of the $d$-derived surface state is occupied; ${ }^{12}$ see PE spectrum in Fig. 1(a). At off-normal emission angles, the $S_{\uparrow}$ peak shifts significantly upon magnetization reversal. From a series of angle-resolved spectra for opposite magnetization directions [cf. Fig. 1(c)], we derived the dispersion around the center of the surface Brillouin zone (SBZ). It is presented in Fig. 2(a), where red and black symbols mark the $S_{\uparrow}$ peak positions for opposite magnetization directions. The energy difference $\Delta \epsilon(\mathbf{k})=E(\mathbf{k}, \mathbf{M})-E(\mathbf{k},-\mathbf{M})$, plotted in Fig. $2(\mathrm{c})$, increases with $\mathbf{k}$, in agreement with the triple-vector product in the Rashba Hamiltonian [see Eq. (1)]. We attribute the separation between peak positions for opposite $\mathbf{M}$ to the 
(a)

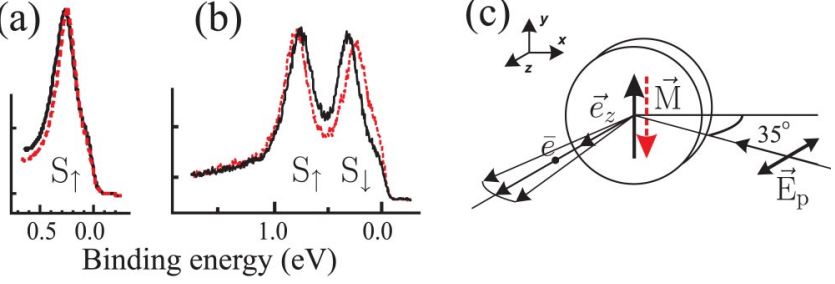

FIG. 1. (Color online) (a) and (b) Angle-resolved PE spectra recorded at $6^{\circ}$ electron-emission angle with respect to the surface normal $\mathbf{e}_{z}$ : (a) Majority-spin surface state $S_{\uparrow}$ of $\operatorname{Gd}(0001)$ (excited with $h \nu=36 \mathrm{eV}$ ); (b) exchange-split two-dimensional states $S_{\uparrow}$ and $S_{\downarrow}$ of $p(1 \times 1) \mathrm{O} / \mathrm{Gd}(0001)(h \nu=45 \mathrm{eV})$. Owing to the Rashba effect, all peak positions change upon magnetization reversal (see text). (c) Experimental geometry.

Rashba interaction of electrons in the majority-spin surface state of $\mathrm{Gd}(0001)$. Although the states for opposite $\mathbf{M}$ do not exist simultaneously, $\Delta \epsilon$ in Fig. 2(c) is equivalent to the Rashba splitting at a nonmagnetic surface, and we shall refer to it as Rashba "splitting" in the following.

The $p(1 \times 1) \mathrm{O} / \mathrm{Gd}(0001)$ surface oxide exhibits an exchange-split pair of occupied two-dimensional bands, ${ }^{11}$ shown as $S_{\uparrow}$ and $S_{\downarrow}$ in Fig. 1(b). Their dispersions are presented in Fig. 2(b), revealing that the peak positions, upon $\mathbf{M}$ reversal, shift into opposite directions and antisymmetrically with respect to the SBZ center. Figure 2(b) shows as well

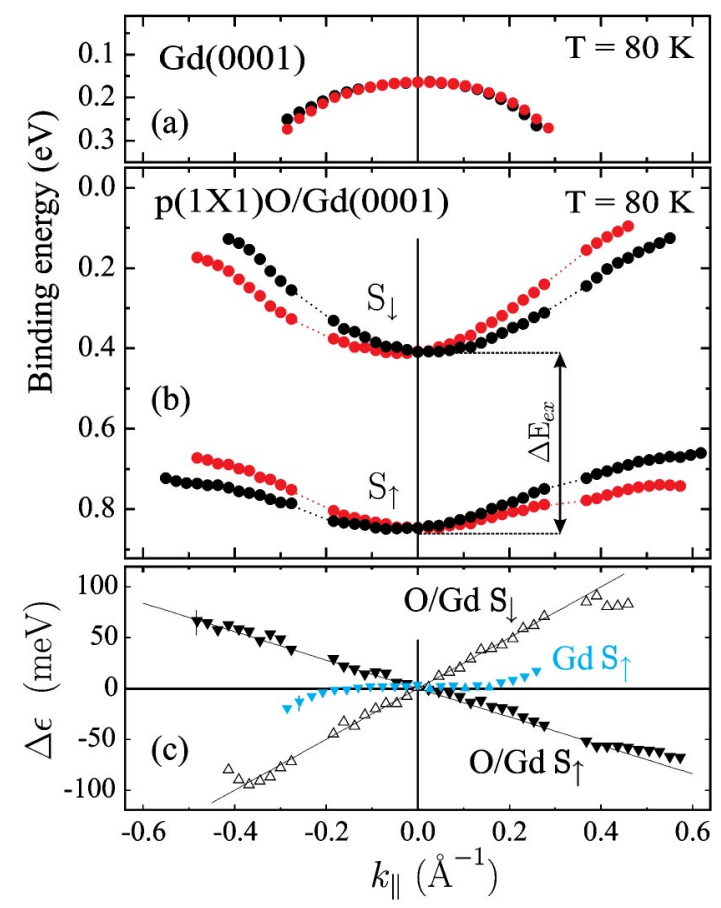

FIG. 2. (Color online) Experimental dispersion of the (a) majority-spin surface state of $\operatorname{Gd}(0001)$ along $\bar{\Gamma}-\bar{M}$ and (b) exchange-split majority spin $\left(S_{\uparrow}\right)$ and minority spin $\left(S_{\downarrow}\right)$ states of $p(1 \times 1) \mathrm{O} / \mathrm{Gd}(0001)$ along $\bar{\Gamma}-\overline{\mathrm{K}}$. The exchange splitting $\Delta E_{e x}$ is $450 \mathrm{meV}$ at the SBZ center. Red and black symbols: opposite magnetization directions. (c) Rashba "splitting" $\Delta \epsilon$ obtained from the data in (a) and (b); $\nabla(\triangle)$ symbols refer to majority (minority) spin states.

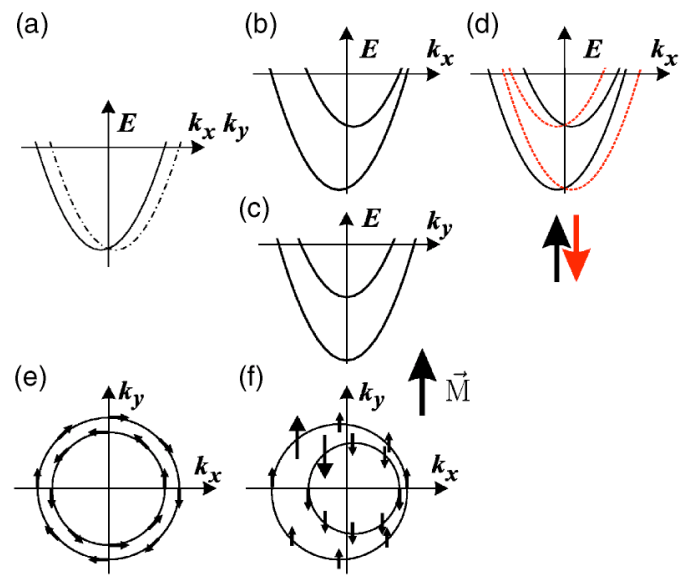

FIG. 3. (Color online) NFE model showing the qualitative difference between the Rashba effect at (a) and (e) a nonmagnetic and (b)-(d) and (f) a FM surface (interface) in the $x, y$ plane, with an in-plane magnetization $\mathbf{M} \| \mathbf{y}$. For details, see text.

that $\Delta E_{e x}$ is much larger than the Rashba splitting $\Delta \epsilon$, i.e., $\mathcal{H}_{R}$ can be regarded as a $k$-dependent (perturbative) energy correction. Thus, basically the spin remains a good quantum number and the spin polarization of the two-dimensional states are discussed either as spin up or down states.

The qualitative differences in appearance between the Rashba effect in magnetic and nonmagnetic systems are highlighted in Fig. 3, using the nearly-free-electron (NFE) model. In nonmagnetic systems, the Rashba interaction splits the spin-degenerate surface state irrespective of the orientation of the in-plane momentum, [see Fig. 3(a)]. In magnetic systems, by contrast, the electron spins are oriented along a quantization axis by exchange interaction-we chose the $y$ axis in Figs. 3(b)-3(d) and 3(f) -and $\mathcal{H}_{R}$ in Eq. (1) becomes anisotropic; the Rashba interaction vanishes for $\mathbf{k} \| \mathbf{s}(\mathbf{k} \| \mathbf{M})$, and only the exchange splitting remains along $\mathbf{k}_{y}$ [see Fig. 3(c)]. Yet, for any other $\mathbf{k}$, both exchange and Rashba splitting become visible, most pronounced along $\mathbf{k}_{x}$ [see Fig. $3(\mathrm{~b})]$. When the magnetization is reversed, the spin $\mathrm{s}$ and with it the triple-vector product in Eq. (1) change sign; the dispersion changes from black to (dotted) red [see Fig. 3(d)]. Hereby the NFE model qualitatively reproduces the experimental behavior shown in Fig. 2(b).

The corresponding Fermi contours are compared in Figs. 3(e) and 3(f). In nonmagnetic systems, the Rashba effect results in an isotropic splitting of the NFE parobola and the Fermi contour splits into a pair of concentric rings. ${ }^{3}$ The spin is, in absence of any other interaction, always oriented perpendicular to the $k$ vector [cf. Fig. 3(e)]. By contrast, in magnetic systems with dominant exchange interaction as in $\mathrm{Gd}(0001)$ and $p(1 \times 1) \mathrm{O} / \mathrm{Gd}(0001)$, it is the exchange splitting that leads to a pair of concentric Fermi contour rings of majority (larger) and minority (smaller) spin electrons. The spin polarization remains practically $100 \%$ as in the limiting case of vanishing Rashba interaction. A finite Rashba interaction simply shifts the majority and the minority Fermi contours into opposite directions [cf. Fig. 3(f)].

For direct comparison, we plot in Fig. 2(c) the Rashba splitting $\Delta \epsilon$ for $S_{\uparrow}$ and $S_{\downarrow}$ of $p(1 \times 1) \mathrm{O} / \mathrm{Gd}(0001)$ together 


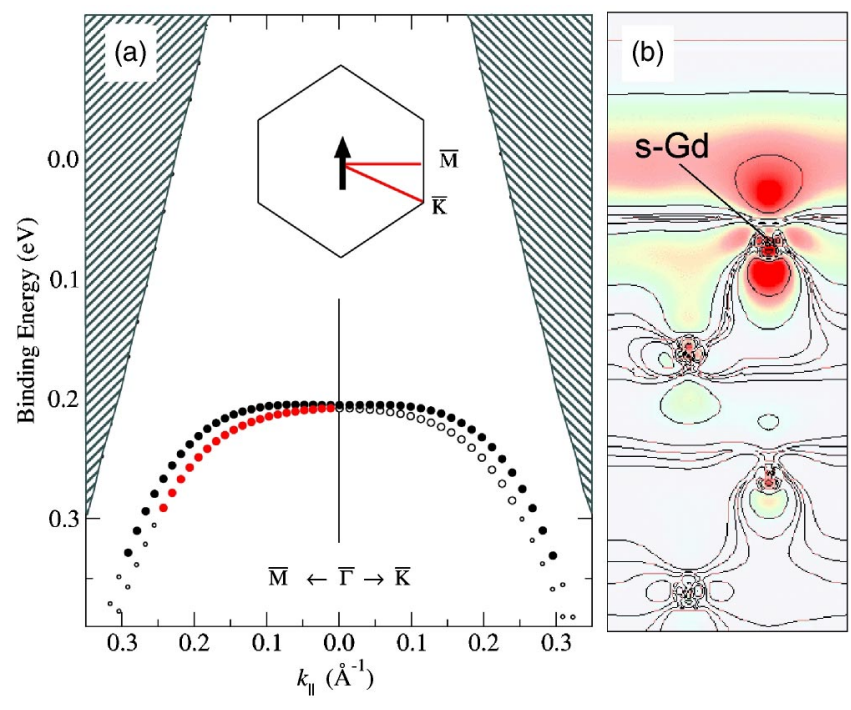

FIG. 4. (Color online) (a) Calculated majority-spin surface-state dispersions of $\operatorname{Gd}(0001)$ along two different high-symmetry directions (shown as red lines) of the magnetic SBZ, given as inset. Hatched areas indicate bulk band regions. (b) Charge-density distribution for $S_{\uparrow}$ at the SBZ center in a plane perpendicular to the surface. The linear color scale ranges from red (high charge density), yellow (medium), green (low), blue (very low), to white (negligible). Isolines: $\log$ scale.

with the one for $S_{\uparrow}$ of $\mathrm{Gd}(0001)$. $\Delta \epsilon$ shows a substantial enhancement (by a factor of 3) upon oxide-layer formation. Moreover, the Rashba parameters of the majority O/Gd interface states and of the majority metal surface state have opposite signs. Obviously, electrons in the interface state are subject to an effective (spatially averaged) crystal electric field that is significantly larger and opposite to the effective field responsible for the Rashba effect at $\mathrm{Gd}(0001)$. We find further that the Rashba "splitting" is opposite for the $S_{\uparrow}$ and $S_{\downarrow}$ states of $p(1 \times 1) \mathrm{O} / \mathrm{Gd}(0001)$, supporting that it is really the Rashba effect that we observed.

To understand the Rashba effect for $\mathrm{Gd}(0001)$ and $p(1 \times 1) \mathrm{O} / \mathrm{Gd}(0001)$ and their strikingly different splittings in a quantitative way, we performed $a b$ initio calculations using density-functional theory (DFT) in the local-density approximation (LDA). ${ }^{13}$ We used the full-potential linearized augmented-plane-wave method in film geometry, ${ }^{14,15}$ where SOC was included self-consistently according to Ref. 16. For a proper description of the $4 f$ electrons, the LDA $+\mathrm{U}$ method was applied. ${ }^{17}$ The Gd surface was simulated by a structurally relaxed 10-layer film embedded in two semi-infinite vacua. A plane-wave cutoff of $k_{\max }=3.8$ a.u. ${ }^{-1}$ was used, and the irreducible part of the SBZ was sampled at $36 \mathbf{k}$ points. It is important to note that the in-plane magnetization lowers the $p 3 m 1$ symmetry of hcp $\operatorname{Gd}(0001)$ to $\mathrm{cm}$ symmetry. Using a pseudohexagonal notation, one can still label the highsymmetry points of the SBZ as $\bar{M}$ and $\bar{K}$, keeping in mind that opposite points of the hexagonal SBZ in Fig. 4(a) are equivalent if one interchanges spin-up and spin-down bands. The magnetization was assumed to point in the direction of the in-plane nearest neighbors.

The calculated dispersion of the states of $\mathrm{Gd}(0001)$ and $p(1 \times 1) \mathrm{O} / \mathrm{Gd}(0001)$, presented in Figs. 4(a) and 5(a), is in

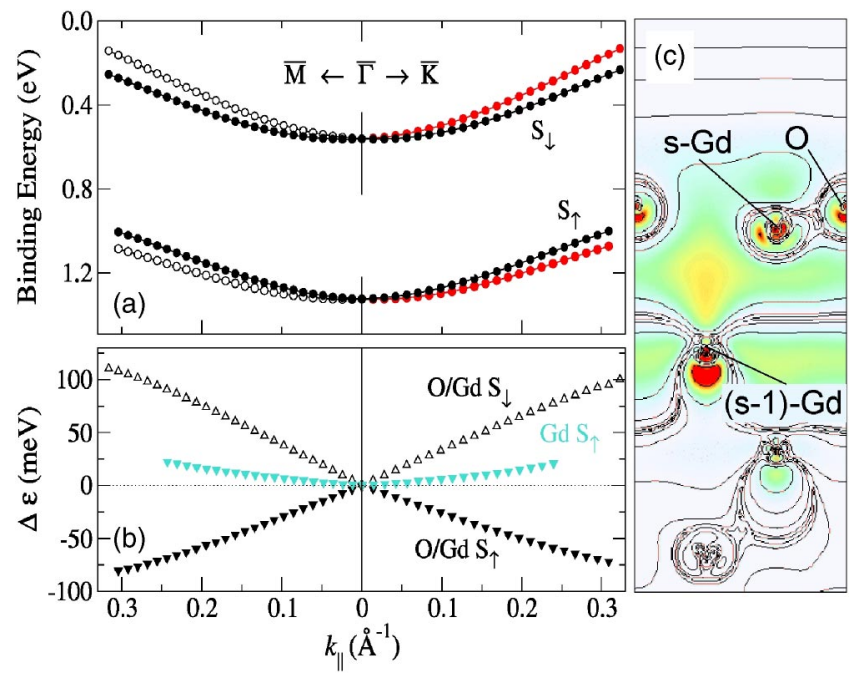

FIG. 5. (Color online) (a) Calculated surface-state dispersions of $p(1 \times 1) \mathrm{O} / \mathrm{Gd}(0001)$ along the high-symmetry directions $\bar{\Gamma}-\overline{\mathrm{M}}$ (left-hand side) and $\bar{\Gamma}-\bar{K}$ (right-hand side) of the SBZ. (b) Rashba "splittings" $\Delta \epsilon$ for majority and minority bands. Symbols and notation are as in Fig. 4.

good agreement with the experimental data (see Fig. 2) even in details ${ }^{18}$ such as the curvature of the states, the size of the Rashba splitting, and the sign reversal of the Rashba constant upon oxide-layer formation [compare Fig. 2(c) with left part (for clean Gd) or right part (for 0/Gd) of Fig. 5(b)].

In Figs. 4(b) and 5(c) the charge-density distributions of the two-dimensional states are displayed in a plane perpendicular to the surfaces. In the calculation of $p(1 \times 1) \mathrm{O} / \mathrm{Gd}(0001)$, the $\mathrm{O}$ atoms were "adsorbed" on the fcc site, and their relaxed positions were found $0.78 \AA$ above the plane of the Gd surface atoms, $s$-Gd. The $s$-Gd layer shows a strong (18\%) outward relaxation upon $\mathrm{O}$ adsorption, while the positions of the inner layers remain almost the same. We note that similar results were obtained for $\mathrm{O}$ adsorbed on the energetically slightly less favorable hcp site. In other words, $p(1 \times 1) \mathrm{O} / \mathrm{Gd}(0001)$ consists of an O/Gd monoxide layer that is quite separated from the nearly unperturbed $\mathrm{Gd}(0001)$ metal.

The charge-density distribution in Fig. 4(b) shows that the $\mathrm{Gd}(0001)$ surface state is localized almost exclusively (to $\sim 89 \%$ ) in the metal surface layer, $s$-Gd. It appears to be almost symmetric with respect to the surface plane, reflecting the rather pure $d_{z^{2}}$ character. ${ }^{17}$ In fact, the charge gradient is small: the calculation yields an admixture of $p_{z}$ character (antisymmetric with respect to the surface plane) with a $d: p$ ratio in the $s$-Gd layer of approximately 8:1 (integrated over the muffin-tin sphere).

Upon oxide-layer formation, the charge distribution of the entire near-surface region changes drastically. Figure 5(c) shows that the two-dimensional state of $p(1 \times 1) \mathrm{O} / \mathrm{Gd}$ is distributed over the whole region between the $\mathrm{O} / \mathrm{Gd}$ surface monoxide layer and the $(s-1)$-Gd layer; hence, this state can be conceived as an interface state. The electronegative $\mathrm{O}$ atom attracts charge not only from the $s$-Gd atom but also from the interface region so that steep charge-density gradi- 
ents arise in both the $s$-Gd layer and the subsurface layer. To identify the main contribution to the Rashba effect, we switched off the SOC in the calculation for individual layers. In this way we found that the $(s-1)$-Gd layer accounts for over $70 \%$ of the total Rashba splitting at $p(1 \times 1) \mathrm{O} / \mathrm{Gd}$. The dominant role of the $(s-1)$-Gd layer becomes obvious from the asymmetric probability distribution in Fig. 5(c); the calculation gives a high admixture of $p_{z}$ character $[d: p \approx 5: 1$, integrated over the $(s-1)$-Gd muffin-tin spheres].

With the present results, we arrive at the following physical picture of the Rashba effect: The charge-density profiles of $\mathrm{Gd}(0001)$ and $p(1 \times 1) \mathrm{O} / \mathrm{Gd}(0001)$ show that there is a close link between the Rashba effect of a two-dimensional state and the asymmetry of the charge distribution. The asymmetry directly reflects the effective electric field an electron experiences in that state; it is responsible for the Rashba effect. The small positive effective electric field in the $s$-Gd layer of $\mathrm{Gd}(0001)$ gives rise to the small Rashba splitting. At $p(1 \times 1) \mathrm{O} / \mathrm{Gd}(0001)$, the rather asymmetric charge distribution of the interface state in the $(s-1)-\mathrm{Gd}$ layer directly reveales the presence of a high effective electric field in this layer. The charge gradient is negative, i.e., opposite to the uncovered metal surface. We are led to conclude that it is this reversed effective electric field in the $(s-1)$-Gd layer that causes the sign change of the Rashba parameter when going from $\operatorname{Gd}(0001)$ to $p(1$ $\times 1) \mathrm{O} / \mathrm{Gd}(0001)$.

In summary, we present here an experimental and theoretical study of the Rashba effect in systems where Rashba and exchange interaction coexist. We demonstrated that the Rashba effect is closely linked to the asymmetry of the wave function that serves as a monitor of the value of the effective potential gradient causing the Rashba effect. We suggest to utilize the spin sensitivity of the Rashba effect in magnetically ordered systems to study two-dimensional spin structures at surfaces and interfaces. The present findings can be of interest for spintronic devices, especially at FM/NM interfaces where Rashba and exchange interaction together control the electron spin.

We gratefully acknowledge experimental support by J. Andersen (MAX-Lab) and R. Püttner (BUS beamline, BESSY). The work in Berlin was supported by BMBF, Contract No. $05 \mathrm{KS} 1 \mathrm{KEC} / 2$, and DFG (SfB-290).
*Corresponding author: Electronic address: starke@physik.fuberlin.de

${ }^{1}$ S. A. Wolf, D. D. Awschalom, R. A. Buhrman, J. M. Daughton, S. von Molnar, A. Y. Chtchelkanova, M. L. Roukes, and D. M. Treger, Science 294, 1488 (2001).

${ }^{2}$ E. I. Rashba, Sov. Phys. Solid State 2, 1109 (1960).

${ }^{3}$ S. LaShell, B. A. McDougall, and E. Jensen, Phys. Rev. Lett. 77, 3419 (1996).

${ }^{4}$ F. Reinert, G. Nicolay, S. Schmidt, D. Ehm, and S. Hüfner, Phys. Rev. B 63, 115415 (2001).

${ }^{5}$ E. Rotenberg and S. D. Kevan, Phys. Rev. Lett. 80, 2905 (1998).

${ }^{6}$ M. Hochstrasser, J. G. Tobin, E. Rotenberg, and S. D. Kevan, Phys. Rev. Lett. 89, 216802 (2002).

${ }^{7}$ J. Henk, A. Ernst, and P. Bruno, Phys. Rev. B 68, 165416 (2003).

${ }^{8}$ I. Zutic, J. Fabian, and S. D. Sarma, Rev. Mod. Phys. 76, 323 (2004).

${ }^{9}$ C. H. Li, G. Kioseoglou, O. van't Erve, A. T. Hanbicki, B. T. Jonker, R. Mallory, M. Yasar, and A. Petrou, Appl. Phys. Lett. 85, 1544 (2004).

${ }^{10}$ The large exchange splitting allows one to avoid the natural limitation in studies of nonmagnetic systems, where the Rashba splitting of a state, to be observed, must be on the order of its width (Ref. 4).

${ }^{11}$ C. Schüssler-Langeheine, R. Meier, H. Ott, Z. Hu, C. Mazumdar, A. Y. Grigoriev, G. Kaindl, and E. Weschke, Phys. Rev. B 60, 3449 (1999).

${ }^{12}$ E. Weschke, C. Schüssler-Langeheine, R. Meier, A. V. Fedorov, K. Starke, F. Hübinger, and G. Kaindl, Phys. Rev. Lett. 77, 3415 (1996).

${ }^{13}$ V. Moruzzi, J. Janak, and A. Williams, Calculated Electronic Properties of Metals (Pergamon, New York, 1978).

${ }^{14}$ E. Wimmer, H. Krakauer, M. Weinert, and A. J. Freeman, Phys. Rev. B 24, 864 (1981).

${ }^{15}$ For program description see http://www.flapw.de

${ }^{16}$ C. Li, A. J. Freeman, H. J. F. Jansen, and C. L. Fu, Phys. Rev. B 42, 5433 (1990).

${ }^{17}$ P. Kurz, G. Bihlmayer, and S. Blügel, J. Phys.: Condens. Matter 14, 6353 (2002).

${ }^{18}$ The exchange splitting is somewhat larger than the experimental value, an overestimation that had been noted before for Gd(0001) (Ref. 17). 